\title{
A NOTE ON ENTIRE FUNCTIONS OF BOUNDED INDEX
}

ANIL K. BOSE

I. Introduction. Let $\left\{f^{(n)}(w) / n !\right\}_{n-0}^{\infty}$ be the sequence of coefficients in the power-series expansion of the entire function $f \neq 0$ about the point $w$. Since the power-series of $f$ about $w$ converges absolutely everywhere in the plane, the sequence $\left\{\left|f^{(n)}(w)\right| / n !\right\}_{n=0}^{\infty}$ must approach zero. Consequently, there exists for each point $w$ a nonnegative integer $n=n(w)$ such that $\left|f^{(n)}(w)\right| / n$ ! is a maximal coefficient. Let $c_{f}(w)$ denote this maximal coefficient and let $I(w)$ be the largest value of $n$ for which $\left|f^{(n)}(w)\right| / n !=c_{f}(w)$.

Definition. If there is an integer $N$ such that $I(w) \leqq N$ for all points $w$, then $f$ is said to be of bounded index and the smallest such integer, which we will denote by $I(f)$, is called the index of $f$.

In view of the above definition, it is clear that

$$
c_{f}(w)=\max \left\{|f(w)|,\left|f^{\prime}(w)\right|,\left|f^{(2)}(w)\right| / 2 !, \cdots,\left|f^{(N)}(w)\right| / N !\right\},
$$

for all points $w$ whenever $f$ is of bounded index with index $\leqq N$.

B. Lepson [1] raised the problem of characterizing entire functions of bounded index. In what follows, we shall give certain answers to Lepson's problem. We will prove the following three properties of entire functions of bounded index:

(i) The function $c_{f}$ satisfies an inequality similar to Harnack inequality for positive harmonic functions. That is, if $\Omega_{N}$ denotes the class of all entire functions of bounded index with index $I(f) \leqq N$ and $D$ be a bounded set, then there exists a positive constant $\mu$, depending only on the diameter of $D$ and the integer $N$, such that the inequality

$$
c_{f}(z) \leqq \mu c_{f}(w)
$$

is true for any two points $z$ and $w$ of $D$ and any member $f$ of $\Omega_{N}$.

(ii) Any sequence $\left\{f_{n}\right\}_{n=1}^{\infty}$ belonging to the family $\Omega_{N}$ is normal if and only if there is a subsequence of the sequence $\left\{c_{f_{n}}\right\}_{x=1}^{\infty}$ which is bounded at a single point. Furthermore, the limit function of any almost uniformly convergent subsequence of $\left\{f_{n}\right\}_{n=1}^{\infty}$ is also of bounded index with index $\leqq N$.

(iii) The index $N$ and the maximal function $c_{f}$ for an exponential function $f$ characterizes the function completely.

II. An inequality for the maximal function $c_{f}$. We begin with the following lemma.

Presented to the Society, April 20, 1968; received by the editors March 4, 1968. 
LEMMA. Let $\Omega_{N}$ denote the class of all entire functions of bounded index with index $\leqq N$. If $f$ be a member of $\Omega_{N}$, then

$$
c_{f}(z) \leqq 2^{N+1} c_{f}(w),
$$

for any two points $z$ and $w$ satisfying $|z-w| \leqq 1 / 2$.

Proof. Let the index of $f$ be $k \leqq N$. We have

$$
f(z)=\sum_{0}^{\infty} \frac{f^{(n)}(w)}{n !}(z-w)^{n},
$$

for all $z$ and $w$ and

$$
\begin{aligned}
& \frac{\left|f^{(j)}(w)\right|}{i !} \leqq c_{f}(w) \\
&=\max \left\{|f(w)|,\left|f^{\prime}(w)\right|,\left|f^{(2)}(w)\right| / 2 !, \cdots,\left|f^{(k)}(w)\right| / k !\right\} \\
&=0,1,2,3, \cdots
\end{aligned}
$$

Since the above power-series is absolutely convergent and the geometric series $\sum_{0}^{\infty}|z-w|^{n}$ is convergent for $|z-w|<1$, it follows that

$$
|f(z)| \leqq c_{f}(w)\left(\sum_{0}^{\infty}|z-w|^{n}\right)=\frac{c_{f}(w)}{1-|z-w|}
$$

for all $z, w$ satisfying $|z-w|<1$.

Now, considering the power-series for the entire function $f^{(j)}$ about $w$ and noting that the series $\sum_{n=j}^{\infty} n(n-1) \cdots(n-j+1) r^{n-j}$, which is obtained by differentiating (term-by-term) the geometric series $\sum_{0}^{\infty} r^{n} j$ times successively, converges to $j ! /(1-r)^{j+1}$, for $|r|<1$, we have

$$
\frac{\left|f^{(j)}(z)\right|}{j !} \leqq \frac{c_{f}(w)}{(1-|z-w|)^{j+1}}, \quad j=0,1,2, \cdots,
$$

for all $z, w$ satisfying $|z-w|<1$. Hence

$$
c_{f}(z) \leqq \frac{c_{f}(w)}{(1-|z-w|)^{k+1}} \leqq 2^{k+1} c_{f}(w) \leqq 2^{N+1} c_{f}(w)
$$

for all $z, w$ satisfying $|z-w| \leqq 1 / 2$. Clearly we can interchange the role of $z$ and $w$ in the inequality (3) and hence the lemma is proved.

THEOREM 1. Let $\Omega_{N}$ denote the class of all entire functions of bounded index with index $\leqq N$. If $D$ be a bounded set, then there exists a positive number $\mu$, depending only on $D$ and $N$ such that the inequality 


$$
c_{f}(z) \leqq \mu c_{f}(w)
$$

is true for any two points $z$ and $w$ of $D$ and any member $f$ of $\Omega_{N}$.

Proof. Let $r$ be the diameter of $D$. There is a positive integer $m$ such that $2 r<m$. Let $z$ and $w$ be any two points of $D$. Then $|z-w| \leqq r$. There is a finite sequence $\left\{z_{p}\right\}_{0}^{k}$ of points with $z_{0}=z, z_{k}=w$ and $k \leqq m$ such that each $z_{p}$ lies on the line segment joining $z$ and $w$ and $\left|z_{p}-z_{p-1}\right|<1 / 2$ for all $p=1,2, \cdots, k$. By the lemma, we have

$$
c_{f}(z)=c_{f}\left(z_{0}\right) \leqq 2^{N+1} c_{f}\left(z_{1}\right) \leqq 2^{2 N+2} c_{f}\left(z_{2}\right) \leqq \cdots \leqq 2^{k N+k} c_{f}\left(z_{k}\right) \leqq 2^{m N+m} c_{f}(w) .
$$

To complete the proof we need only to choose $\mu=2^{m N+m}$.

Definition. A sequence $\left\{f_{n}\right\}_{1}^{\infty}$ of entire functions is said to be almost uniformly convergent in the plane, if it converges uniformly on every compact subset of the plane.

As an application of the inequality (4), we will now prove the following theorem regarding the normality of any subfamily of $\Omega_{N}$

TheOREM 2. Let $\left\{f_{n}\right\}_{n=1}^{\infty}$ be a sequence such that for each positive integer $n, f_{n}$ is in $\Omega_{N}$.

(i) $\left\{f_{n}\right\}_{1}^{\infty}$ is normal, i.e., there exists a subsequence $\left\{f_{k}^{*}\right\}_{1}^{\infty}$ of $\left\{f_{n}\right\}_{1}^{\infty}$ such that $\left\{f_{k}^{*}\right\}_{1}^{\infty}$ is almost uniformly convergent in the plane to an entire function $g$, if and only if there is a subsequence $\left\{c_{f_{k}^{*}}\right\}_{1}^{\infty}$ of the sequence $\left\{c_{f_{n}}\right\}_{1}^{\infty}$ which is bounded at a single point.

(ii) If $\left\{f_{n}\right\}_{1}^{\infty}$ is almost uniformly convergent in the plane to an entire function $g$, then $g$ is of bounded index with index $\leqq N$.

Proof. Suppose that $\left\{f_{n}\right\}_{1}^{\infty}$ is normal and $\left\{f_{k}^{*}\right\}_{1}^{\infty}$ be a subsequence which is almost uniformly convergent in the plane to an entire function $g$. Let $w$ be a point and $\epsilon$ be a positive number. Since the sequence $\left\{f_{k}^{*(j)}(w) / j !\right\}_{k=1}^{\infty}$ converges to $g^{(j)}(w) / j$ ! for all $j=0,1,2,3, \cdots$, there is a positive integer $k_{j}$ such that

$$
\left|f_{k_{j}+n}^{*(j)}(w) / j !-g^{(j)}(w) / j !\right|<\epsilon \text { or }\left|f_{k_{j}+n}^{*(j)}(w)\right| / j !<\left(\left|g^{(j)}(w)\right| / j !\right)+\epsilon,
$$

for all $n, j=0,1,2,3, \cdots$. Let $m=\operatorname{Max}\left\{k_{0}, k_{1}, k_{2}, \cdots, k_{N}\right\}$, then

$$
\left|f_{m+n}^{*(j)}(w)\right| / j !<\left(\left|g^{(j)}(w)\right| / j !\right)+\epsilon
$$

for all $n$ and for $j=0,1,2, \cdots, N$. Since each $f^{*}{ }_{m+n}$ is in $\Omega_{N}$, we have from (5)

$$
c_{f_{m+n}^{*}}(w)<c_{0}(w)+\epsilon \text {, for all } n \text {. }
$$

This shows that the subsequence $\left\{c_{f_{k}^{*}}\right\}_{k=1}^{\infty}$ of the sequence $\left\{c_{f_{n}}\right\}_{1}^{\infty}$ is bounded at the point $w$. (In fact, we have proved that the subse- 
quence $\left\{c_{f_{k}^{*}}\right\}_{1}^{\infty}$ is bounded at each point of the plane.) Conversely, let $w$ be a point and $\left\{c_{k}^{*}\right\}_{k-1}^{\infty}$ be a subsequence of $\left\{c_{f_{k}}\right\}_{i}^{\infty}$ such that $\left\{c_{f_{k}^{*}}\right\}_{1}^{\infty}$ is bounded at $w$. Let $S$ be a compact set of the plane. We can find a bounded set $S$ such that $w \in S$ and $S \subseteq S$. There is a positive number $M$ such that $c_{f_{k}^{*}}(w) \leqq M$ for all $k$. By Theorem 1 , there is a positive number $\mu=\mu(\hat{S})$, depending only on $S$ (hence on $S$ ) and $N$ such that

$$
c_{f k}^{*}(z) \leqq \mu(\hat{S}) c_{j k}^{*}(w) \leqq \mu(\hat{S}) M
$$

for all $k$ and each $z$ in $S$. Since $\left|f_{k}^{*}(z)\right| \leqq c_{f_{k}^{*}}(z)$, for all points $z$, it follows that

$$
\left|f_{k}^{*}(z)\right| \leqq \mu(S) \boldsymbol{M}
$$

for each $z$ in $S$ and for each $k=1,2,3, \ldots$. Hence the sequence $\left\{f_{k}^{*}\right\}_{1}^{\infty}$ is uniformly bounded on compact subsets of the plane. Therefore, by Vitali's Theorem, there is a subsequence $\left\{f_{p}^{* *}\right\}_{p-1}^{\infty}$ of $\left\{f_{k}^{*}\right\}_{1}^{\infty}$ such that $\left\{f_{p}^{* *}\right\}_{i}^{\infty}$ is almost uniformly convergent in the plane to an entire function $g$. Since $\left\{f_{n}^{* *}\right\}_{1}^{\infty}$ is also a subsequence of $\left\{f_{n}\right\}_{1}^{\infty}$, the proof of part (i) is complete.

To prove part (ii), suppose that $\left\{f_{n}\right\}_{i}^{\infty}$ is almost uniformly convergent in the plane to the entire function $g$. Our proof will be complete if we can show that

$$
\begin{aligned}
\boldsymbol{M}_{\theta}(z) & =\max \left\{|g(z)|,\left|g^{\prime}(z)\right|,\left|g^{(2)}(z)\right| / 2 !, \cdots,\left|g^{(N)}(z)\right| / N !\right\} \\
& =c_{\theta}(z)
\end{aligned}
$$

for all points $z$. Let $z$ be a point. If possible, let $M_{\theta}(z)<c_{\theta}(z)$, then $c_{g}(z)=\left|g^{(p)}(z)\right| / p$ ! for some $p>N$. Let $\epsilon$ be a positive number such that $2 \epsilon<\delta$, where $0<\delta=c_{0}(z)-M_{\theta}(z)$. Since the sequence $\left\{f_{n}^{(j)}(z) / j !\right\}_{n=1}^{\infty}$ converges to $g^{(j)}(z) / j$ !, for all $j$, arguing similarly as in (5), there is a positive integer $m$ such that

$$
\left|f_{m+n}^{(j)}(z)\right| / j !<\left(\left|g^{(j)}(z)\right| / j !\right)+\epsilon
$$

for all $\boldsymbol{x}$ and for $j=0,1,2,3, \cdots, N$. Since each $f_{k}$ is in $\Omega_{N}$, we have

$$
c_{f_{m+m}}(z)<M_{0}(z)+\epsilon
$$

for all $n$. Again there is a positive integer $l$ such that

$$
\left|g^{(p)}(z)\right| / p !<\left(\left|f_{l+n}^{(p)}(z)\right| / p !\right)+\epsilon
$$

for all $n$. Hence if $n_{1}$ and $n_{2}$ be two in tegers such that $m+n_{1}=l+n_{2}=q$, say, then from (6) and (7), we get 


$$
\begin{aligned}
M_{0}(z)+\delta & =c_{0}(z)=\left|g^{(x)}(x)\right| / p ! \\
& <\left(\left|f_{0}^{(z)}(z)\right||p|\right)+\epsilon \leqq c_{g}(z)+\epsilon \\
& <M_{0}(z)+2 \epsilon<M_{0}(z)+\delta .
\end{aligned}
$$

This implies a contradiction, hence

$$
c_{0}(z)=\max \left\{|g(z)|,\left|g^{\prime}(z)\right|,\left|g^{(z)}(z)\right| / 2 !, \cdots,\left|g^{(N)}(z)\right| / N !\right\}
$$

for all points $z$. In other words $g$ is of bounded index with index $\leqq N$.

1II. The index of exponential functions. In what follows we will describe a characteristic property of exponential functions in terms of their indices.

THEOREM 3. Let $f \not \equiv 0$ be an entire function of bounded index with index $N . f$ is an exponential of the form $e^{a z+b}(a, b$, constants) if and only if $c_{f}(z)=\left|f^{(N)}(z)\right| / N$ ! for all points $z$.

Proof. Suppose that $c_{f}(z)=\left|f^{(N)}(z)\right| / N$ ! for all points $z$, where $N$ is the index of $f$. This means that

$$
\left|f^{(j)}(z)\right| / j ! \leqq\left|f^{(N)}(z)\right| / N !
$$

for all points $z$ and $j=0,1,2,3, \cdots$ Also $f^{(N)}$ cannot vanish anywhere in the plane. For, $f^{(N)}(z)=0$, for some point $z$, implies by (8) that $f^{(j)}(z)=0$ for all $j=0,1,2,3, \cdots$. Hence $f$ vanishes identically in the entire plane which contradicts our assumption. Therefore $f / f^{(N)}$ is an entire function. Again from (8)

$$
\left|\frac{f(z)}{f^{(N)}(z)}\right|=\frac{1}{N !} \frac{|f(z)|}{\left|f^{(N)}(z)\right| / N !} \leqq \frac{1}{N !} \text { for all points } z,
$$

which implies that $f / f^{(N)}$ is bounded and hence a constant, $a_{1}$, say. So that we have

$$
f=a_{1} f^{(N)}
$$

Clearly $a_{1} \neq 0$. Similarly

$$
f^{\prime}=a_{2} f^{(N)} \text {, where } a_{2} \text { is some constant. }
$$

Therefore $f^{\prime}=a f$, where $a=a_{2} / a_{1}$. Hence there is a constant $c \neq 0$, such that $f(z)=c e^{a z}$ or $f(z)=e^{a z+b}$ for all points $z$, where $c=e^{b}$. Conversely, suppose that $f(z)=e^{a s+b}$, for all points 8 , where $a, b$ are constants. If $|a| \leqq 1$, then it is easy to see that the index of $f$ is 0 and $c_{f}(z)=|f(z)|$ $=\left|f^{(0)}(z)\right| / 0$ !, for all points $z$. So let $|a|>1$ and $N=[|a|]=$ the greatest integer less than $|a|$. We have 


$$
\frac{|a|}{N+1} \leqq 1<\frac{|a|}{N} \text {. }
$$

$$
\begin{aligned}
& \left|f^{(j)}(z)\right| / j !=\frac{|a|^{j}}{j !}\left|e^{a z+b}\right|, \text { for all } j \text { and all } z, \\
& |f(z)|<\left|f^{\prime}(z)\right|<\left|f^{(2)}(z)\right| / 2 !<\cdots<\left|f^{(N)}(z)\right| / N ! \\
& \left|f^{(N+n)}(z)\right| /(N+n) !=\frac{|a|^{N+n}}{(N+n) !}\left|e^{a z+b}\right| \\
& \leqq \frac{|a|^{N}}{N !}\left|e^{a z+b}\right|=\frac{\left|f^{(N)}(z)\right|}{N !}
\end{aligned}
$$

for all points $z$ and all $n=1,2,3, \ldots$. It is clear from the above four inequalities that the index of $e^{a z+b}$ is $N=[|a|]$ and

$$
c_{f}(z)=\left|f^{(N)}(2)\right| / N !
$$

for all points $z$. This completes the proof.

The following corollary follows immediately from Theorem 3.

COROLLARY. If an entire function $f$ is of bounded index with index zero, then it is an exponential of the form $e^{a z+b}$, where $a$ and $b$ are constants and $|a| \leqq 1$.

\section{REFERENCES}

1. B. Lepson, Differential equations of infinite order, hyperdirichlet series and entire functions of bounded index, Lecture Notes, Summer Institute on Entire Functions, University of California, La Jolla, California, 1966.

2. Fred Gross, Entire functions of bounded index, Proc. Amer. Math. Soc. 18 (1967), 974-980.

Clemson University 